\title{
GENRE

\section{Dénoncer les féminicides des femmes autochtones aux États-Unis et au Canada}

La bande dessinée Deer Woman d'Elizabeth LaPensée, une super-héroïne autochtone éco-féministe?

\section{Aurélie Journée-Duez}

\section{OpenEdition}

Journals

Édition électronique

URL : http://journals.openedition.org/ges/726

DOI : $10.4000 /$ ges.726

ISSN : 2431-6563

Éditeur

Presses universitaires de Bordeaux

Référence électronique

Aurélie Journée-Duez, « Dénoncer les féminicides des femmes autochtones aux États-Unis et au

Canada ", Genre en séries [En ligne], 10 | 2019, mis en ligne le 01 décembre 2019, consulté le 18 février 2021. URL : http://journals.openedition.org/ges/726 ; DOI : https://doi.org/10.4000/ges.726

Ce document a été généré automatiquement le 18 février 2021.

\section{cc) (†)}

La revue Genre en séries est mise à disposition selon les termes de la Licence Creative Commons Attribution - Pas d'Utilisation Commerciale - Pas de Modification 4.0 International. 


\section{Dénoncer les féminicides des femmes autochtones aux États-Unis et au Canada}

La bande dessinée Deer Woman d'Elizabeth LaPensée, une super-héroïne autochtone éco-féministe?

\section{Aurélie Journée-Duez}

\section{Introduction}

«The central issue that confronts American Indian women throughout the hemisphere is survival, literal survival, both on a cultural and biological level $»^{1}$.

1 Si le monde de la bande dessinée occidentale a mis en scène durant ces dernières années des personnages d'origines autochtones ${ }^{2}$, les peuples que ces derniers incarnent se sont eux-mêmes représentés bien avant. En effet, depuis les années 1940, des artistes autochtones des États-Unis et du Canada explorent l'univers de la bande dessinée, en produisant ponctuellement des dessins satiriques pour des revues, des journaux et en réalisant également des bandes dessinées à part entière ${ }^{3}$, avec un héros principal autochtone. Ces artistes parodient et dénoncent le quotidien des peuples autochtones aux États-Unis et au Canada, et/ou inventent un monde extraordinaire au sein duquel ils ont toute leur place. C'est notamment ce qu'a mis en exergue l'exposition « Comic Art Indigène: Where Comics and the Indigenous Meet» (au National Museum of the American Indian à Washington, D.C., puis au Museum of Indian Arts and Culture à Santa Fe, Nouveau-Mexique, entre 2008 et 2009).

Depuis ces deux dernières années, la bande dessinée autochtone nord-américaine connaît un nouvel essor, mêlant formes et motifs ancestraux à des innovations stylistiques allant au-delà de la dichotomie tradition/modernité (Marie Mauzé, 2018). Ainsi, la création de l'Indigenous Comic Con en 2016 à Albuquerque (Nouveau-Mexique, 
États-Unis), évènement qui réunit des artistes parmi les plus prometteu.r.ses de leur génération ${ }^{4}$, souligne la vitalité et la puissance de ce mouvement en pleine transformation qui n'hésite pas à promouvoir, grâce à l'art, des revendications politiques et sociales faisant écho à l'actualité. Outre l'affirmation de l'existence de pratiques artistiques autochtones contemporaines novatrices, le monde de la bande dessinée crée ainsi une occasion supplémentaire de voir émerger des figures d'artistes femmes, engagées et militantes. Nous pouvons citer par exemple Arigon Starr (Kickapoo, Oklahoma), la "Native American Diva» selon ses propres termes. Dessinatrice et créatrice de Super Indian, elle revisite le comic book Superman en mettant en scène un personnage autochtone sur un registre humoristique.

Récit à la fois brillant et sombre d'Elizabeth LaPensée ${ }^{5}$, Deer Woman: A Vignette (2015) constitue un exemple récent de l'appropriation du monde de la bande dessinée par des artistes femmes autochtones. Dessinée dans cette édition par l'artiste Jonathan R. Thunder ${ }^{6}$, cette super-héroïne sans nom (seul le titre de la bande dessinée nous donne une indication) a ensuite été mise en scène en collaboration avec Weshoyot Altrive ${ }^{7}$ dans l'ouvrage collectif Deer Woman: An Anthology (2017) ${ }^{8}$ aux côtés d'autres œuvres comportant une Femme Cerf (Deer Woman).

4 L'objectif principal de cet article est ainsi d'interroger la notion de «super-hérö̈ne » à l'aune de représentations autochtones engagées dans la bande dessinée, aux États-Unis et au Canada' ${ }^{9}$. Plus spécifiquement, il s'agit de voir en quoi la revitalisation du mythe traditionnel de la Femme Cerf dans l'œuvre d'Elizabeth LaPensée, interroge à la fois l'identité en termes de sexe et de genre, ainsi que la culture et la place des femmes autochtones « marchant entre deux mondes ${ }^{10}$ » (Fitzgerald, 2006). Cette relecture de la Femme Cerf prend une position radicale face au problème social et sociétal ${ }^{11}$ des féminicides ${ }^{12}$ en Amérique du Nord, qui constituent un génocide selon le rapport final de "l'Enquête nationale sur les femmes et les filles autochtones disparues et assassinées » (ENFFADA), paru en juin $2019^{13}$.

5 Influencés notamment par les travaux de l'essayiste féministe française Françoise d'Eaubonne (1920-2005) et son approche de l'éco-féminisme, nous postulons que la figure de Deer Woman, être mi-femme mi-cerf, pourrait constituer une représentation allégorique dénonçant la domination masculine sur les femmes (autochtones) et plus largement sur l'environnement. Les théories dites « éco-féministes » naissent dans les années 1970, lorsque l'essayiste féministe française précitée publie en 1974 son ouvrage devenu célèbre: Le féminisme ou la mort, dans lequel elle déclare : "Le rapport de l'homme à la nature est plus que jamais, celui de l'homme à la femme " (D'Eaubonne, 1978 : 15). Par ces mots, elle met en évidence que l'exploitation de la Terre par l'homme s'inscrit dans la logique patriarcale qui tend à dominer les femmes et toutes autres espèces. ${ }^{14}$.

6 Notre article s'inscrit dans le cadre d'une recherche doctorale en anthropologie et en histoire de l'art, portant sur le rôle et la place du médium photographique dans les pratiques contemporaines des artistes autochtones femmes et queer aux États-Unis et au Canada. Plus spécifiquement, il s'agit de voir en quoi l'utilisation récurrente de la photographie dans des œuvres de médiums différents participe depuis la fin des années 1960 et du début des années 1970 de revendications identitaires, politiques et sociales dans une perspective à la fois intersectionnelle et décoloniale ${ }^{15}$.

7 À partir d'une lecture culturelle, politique et sociale de la figure de la Femme Cerf, nous étudierons tout d'abord les caractéristiques formelles et stylistiques de Deer Woman et 
des mythes fondateurs qui en sont à l'origine (I.). Cette première étape nous permettra de comprendre en quoi le personnage de la Femme Cerf dénonce les féminicides dont sont victimes les femmes autochtones aux États-Unis et au Canada depuis maintenant plusieurs décennies, et met en évidence les réalités sociales et sociétales auxquelles celles-ci doivent faire face, fruits désastreux de la colonisation, du racisme et de l'impunité d'État qui font leur quotidien (II.). Enfin, nous nous demanderons en quoi la super-héroïne Deer Woman pourrait laisser entrevoir l'émergence d'une figure autochtone éco-féministe ${ }^{16}$ dans l'univers de la bande dessinée (III.).

\section{«Indigéniser ${ }^{17}$ » la bande dessinée : rendre visible les femmes et les cultures autochtones dans la société contemporaine nord-américaine}

8 La bande dessinée Deer Woman: A Vignette (2015) se compose de vignettes noires et blanches (à l'exception de quelques taches rouges), et présente un style sobre mais efficace dont les traits vifs et visibles participent d'une atmosphère sombre. Chaque dessin a été réalisé par Jonathan $R$. Thunder, artiste peintre plurimédia résidant à Duluth, dans le Minnesota, qui déclare au sujet de son travail de peintre :

Mes peintures explorent des thèmes personnels liés à l'identité et au dialogue interne entre la narration et les personnages. Je représente des personnages expressifs dont les émotions et les pensées se manifestent de façon viscérale dans leur aspect physique. Les corps de mes sujets apparaissent souvent fragmentés, bestiaux, ou partiellement dissimulés. Mon art est comme un album qui enregistre une identité en mutation. À travers mes sujets, je peux exagérer les méchants et les héros qui composent la vision que j'ai de moi-même et du monde. Mon identité culturelle s'imprègne de mon identité de citadin. Mes tableaux sont des vignettes par nature, des aperçus de moments d'une histoire qui ne commence ni se termine sur la toile ${ }^{18}$.

Jonathan R. Thunder conçoit donc ses tableaux comme des vignettes ${ }^{19}$, c'est-à-dire des représentations et des scènes conçues dans un espace-temps spécifique. Cette esthétique est ici transférée dans le média de la bande dessinée. Dans Deer Woman, chaque image illustre un instant précis de l'histoire que narre Elizabeth LaPensée, celle de la transformation de l'héroïne. La deuxième page de cette bande-dessinée s'ouvre sur un personnage en train de courir, dont le corps n'est visible qu'à moitié, mais dont le mouvement des jambes, des coudes et des cheveux indique aux lecteurs que quelqu'un.e s'élance dans une aventure, dont le schéma narratif est a priori assez conventionnel s'agissant du thème des super-héroïn.e.s en particulier : c'est à la suite d'un événement spécifique qu'un personnage banal acquiert ses pouvoirs. Le récit de Deer Woman, être mi-femme, mi-cerf, peut être découpé en six épisodes: la vie quotidienne du personnage principal (une jeune lycéenne qui se rend à une fête avec un camarade), le traumatisme fondateur (Lainé, 2011) bouleversant la vie du personnage dit «ordinaire» (le viol par son ami qui lui a en réalité menti sur la tenue d'une fête), la transformation et l'acquisition des pouvoirs, le moment de doute et d'introspection qui en découle (remémoration de la vie passée), l'utilisation de ces nouveaux pouvoirs, et enfin une ouverture vers l'avenir faite par le personnage principal. La force du personnage de la Femme Cerf provient d'une agression sexuelle, d'un viol. Il y a là une certaine ambiguïté dans la mise en récit de l'empowerment des femmes, qui n'est pas propre à Deer Woman en particulier mais se retrouve dans 
plusieurs autres figures de fictions féminines et féministes, telles que Katniss Everdeen dans Hunger Games (Oliver, 2016).

Les dessins de Jonathan $\mathrm{R}$. Thunder suggèrent cette transformation plus qu'ils ne la représentent, et traduisent ainsi en images la complexité interne des changements qui s'opèrent dans l'être de la jeune femme qui les subit. L'artiste juxtapose au regard humain celui d'un cerf (page 6) après que le viol a eu lieu; il représente l'ombre (en forme de cerf) de la femme marchant dans la rue, puis les sabots de la jeune femme qui se transforme en Femme Cerf alors qu'une seconde agression sexuelle est sur le point d'avoir lieu (page 9). Il montre que la transformation s'accomplit en représentant les empreintes des pattes de la Femme Cerf dans le sang de l'agresseur (page 9 également) - le rouge, seule couleur utilisée, n'intervient que quatre fois dans la bande dessinée, pour représenter le sang et une coccinelle située au début et à la fin du livre. Jonathan R. Thunder se souvient: "La légende de la Femme Cerf m'a été racontée dans ma famille comme un conte effrayant. J'apporte ici de la beauté et de la féminité au personnage classique $»^{20}$.

11 Si Deer Woman apparaît bien comme le récit d'une super-héroïne, Elizabeth LaPensée n'en est en réalité par tout à fait l'auteure, mais plutôt l'interprète. En effet, la bande dessinée Deer Woman ("Waawaashkeshi kwe» en langue anishinaabe ou «Thauca Winyan» en lakota) s'inspire d'un mythe traditionnel autochtone partagé par les peuples Anishinaabe, Potawatomi, Creek, Omaha, Ponca, mais aussi Lakota, comme l'explique l'anthropologue Marla Powers (Université Seton Hall, New Jersey) :

Un autre exemple de symbolisme féminin est représenté par le personnage mythologique d'Anukite, qui joue un rôle important dans les affaires surnaturelles chez les Oglala. Dans la cosmologie Ite (Visage) est très belle et mariée à Tate (Vent). Ite a une histoire avec le Soleil, qui est alors marié à la Lune. À cause de son infidélité, Ite est punie et dotée d'un visage laid. D'un côté du visage, elle est toujours belle, et de l'autre elle est laide. Elle devient ensuite Anukite (Visage Double), qui, d'après Powers, "apparaît aux hommes dans des visions et dans le monde réel sous la forme d'un cerf ou de deux femmes cerfs, l'une blanche et l'autre noire. Les deux visages de Visage Double et les deux Femmes Cerfs représentent respectivement les comportements sexuels conformes et impropres. Les hommes deviennent dérangés ou fous quand ils ont des relations sexuelles (impropres) avec des femmes cerfs ${ }^{21}$. Les femmes qui rêvent d'Anukite ont des pouvoirs inhabituels pour séduire les hommes. Ces femmes étaient considérées comme wakan (sacrées). Les Oglala racontent que si un homme rencontre une femme seule dans les bois ou dans la prairie, il doit l'éviter, car elle pourrait être une femme cerf. Le mythe attribue au cerf une odeur particulière dans le sabot, qui va devenir un parfum agréable quand le cerf va se transformer en femme. Le parfum agit ensuite comme un médicament et ensorcèle les hommes. Parfois, rien que le fait de souhaiter faire l'amour avec une femme cerf peut être fatal. Les femmes cerfs apparaissent souvent comme de belles créatures qui, après avoir séduit les hommes pour qu'ils aient des relations sexuelles incorrectes avec elles, se transforment en cerfs et s'en vont. Après quoi l'homme devient fou ou meurt ${ }^{22}$.

Dans ce mythe autochtone, la Femme Cerf a le pouvoir de tuer les hommes qui lui apparaissent comme potentiellement dangereux. Elle est donc un personnage dont la force et la puissance effraient. Redoutée des hommes, la Femme Cerf est le pendant de la femme victime, elle est celle qui peut assurer sa propre défense. Traditionnellement, les sociétés autochtones aux États-Unis et au Canada sont matriarcales, la femme est au centre de l'organisation sociétale et y occupe donc une place fondamentale. Ainsi, beaucoup de peuples autochtones partagent des récits, des contes, des mythes, mettant 
en scène le personnage d'une femme forte, qui détient des pouvoirs spécifiques, parfois déterminants dans les relations entre hommes et femmes ${ }^{23}$. C'est notamment le cas de Deer Woman, de Double Woman, ou de Spider Woman, dans la culture diné (navajo) notamment ${ }^{24}$.

13 À travers la bande dessinée Deer Woman: A Vignette, Elisabeth LaPensée, Jonathan R. Thunder puis Weshoyot Altrive, mettent en récit et représentent un personnage fort et résilient dont les femmes autochtones pourraient s'inspirer, à rebours des représentations qui n'insistent que sur leur position de victimes. Ainsi, d'après Weshoyot Altrive :

Il y a beaucoup de souffrance et beaucoup d'idéaux féminins brisés dans la culture autochtone, en particulier de nos jours. Et j'espère que ce j'ai fait avec ce livre [...], c'est prendre cette figure perdue et brisée, quasiment mythologique, et la confronter à des questions modernes. Afin que ce livre touche à de vrais problèmes et touche de vraies femmes autochtones ${ }^{25}$.

Dans Deer Woman, la super-héroïne se transforme en cerf en cas de danger imminent, et lutte, jusqu'à ce que mort s'ensuive, contre ses agresseurs. La transformation en un animal de sexe biologique masculin (il s'agit bien d'un cerf et non d'une biche, les dessins représentant des bois sur le haut de la tête du personnage en témoignent) met en évidence le caractère non seulement transgenre de la super-héroïne mise en scène, mais plus encore, le fait que ce personnage est trans-espèce et rompt la dichotomie entre l'être humain et l'animal. Il s'inscrit ainsi dans la continuité des figures au centre de plusieurs récits traditionnels autochtones en Amérique du Nord. Nous pouvons citer par exemple le mythe autochtone de White Buffalo Calf Woman, messagère du Créateur, qui après avoir apporté la pipe originelle sacrée au peuple lakota, ainsi que les sept cérémonies qui l'accompagnent, se transforme en bisonne blanche ${ }^{26}$.

Le choix d'Elizabeth LaPensée de mettre en scène une femme capable de se défendre et de tuer ses agresseurs, tout en revitalisant un mythe traditionnel, ne répond pas seulement à des considérations scénaristiques. La bande dessinée Deer Woman a en effet été créée dans le contexte spécifique des féminicides de femmes autochtones en cours aux États-Unis et au Canada ${ }^{27}$ depuis les années 1980. La transmission de cette histoire par le prisme de la bande dessinée est importante pour les communautés autochtones puisqu'elle attire l'attention sur les disparitions et les meurtres de femmes autochtones, aussi bien en Colombie-Britannique (Canada) que dans la région des Grands Lacs (États-Unis) ${ }^{28}$.

\section{Du mythe à la réalité : dénoncer les féminicides des femmes autochtones en Amérique du Nord}

Deer Woman, œuvre de fiction, s'inspire clairement des disparitions et assassinats de femmes autochtones, une situation bien réelle à laquelle chacun.e.s tentent d'apporter des réponses, par l'art, mais aussi par des pratiques de développement personnel et physique (ce que nous verrons dans notre dernière partie). Dès les premières pages de Deer Woman, Elizabeth LaPensée écrit ainsi :

[Deer Woman] est un appel à prendre conscience et à reconnaître que plus de 1000 femmes autochtones de tous âges sont portées disparues ou ont été assassinées. Cela se passe le long de la région des Grands Lacs où de jeunes femmes et beaucoup d'autres sont impliquées dans des réseaux de trafic sexuel par bateau. [Deer Woman] nous invite à prendre position pour changer le fait que les femmes autochtones 
sont les plus susceptibles de subir une attaque sexuelle sur la Grande Ile de la

Tortue, et pour que soit reconnu le manque de droits ${ }^{29}$.

17 Autrement dit, Deer Woman apparaît ici comme un personnage-métaphore qui incarne la réalité des violences sexuelles auxquelles sont confrontées au quotidien les femmes autochtones au Canada. Car les cas de féminicides en Amérique du Nord ne constituent pas des cas isolés. Pour prendre la mesure de l'ampleur de cette véritable pandémie, il semble important de se baser sur quelques chiffres. Ainsi, l'Indian Law Resource Center rapporte que :

Aux États-Unis, la violence contre les femmes autochtones atteint des niveaux sans précédent sur les terres tribales et dans les villages autochtones d'Alaska. Plus de quatre femmes autochtones sur cinq ont subi des actes violents, et plus d'une femme sur deux est victime de violences sexuelles. Les femmes autochtones en Alaska font face au taux le plus élevé d'agressions sexuelles et les enquêtes concluent à un taux de violence domestique dix fois supérieur à celui du reste des États-Unis. Bien que les données disponibles soient limitées, le nombre de femmes autochtones disparues ou assassinées et le manque de réponse du gouvernement fédéral sont alarmants, aussi bien pour les femmes autochtones, pour les gouvernements tribaux ou pour les communautés. Sur certaines réserves, les assassinats de femmes autochtones sont dix fois plus nombreux que sur l'ensemble $\mathrm{du}$ territoire national ${ }^{30}$.

18 Le Canada n'est pas épargné, bien au contraire. Comme l'a montré Emmanuelle Walter dans son ouvrage Sœurs Volées, les femmes autochtones sont victimes de féminicides depuis plusieurs décennies, notamment en Colombie-Britannique le long de la route 16, désormais tristement connue sous le nom de «Highway of Tears» («Route des Larmes » en français). Les chiffres sont éloquents : «Entre 1980 et 2012, 1181 femmes autochtones ont disparu ou ont été assassinées [...] alors qu'elles ne représentent que 4\% des femmes au Canada [...] Rappelons-nous que, proportionnellement, 1181 femmes autochtones représentent environ 30000 femmes canadiennes ou 55000 femmes françaises $»^{31}$. La plupart de ces cas de disparition et de meurtre, classés sans suite ou irrésolus, sont liés à des situations sociales d'extrême précarité, de vulnérabilité (pour reprendre les termes d'Emmanuelle Walter), dans des contextes de racisme et de sexisme banalisés par une société patriarcale à majorité blanche. Fanny Wilde, avocate algonquine membre de la Commission d'Enquête sur les Femmes Disparues et Assassinées $^{32}$ a par ailleurs recueilli des témoignages auprès de 70 familles avec lesquelles elle a travaillé, mettant en exergue un autre type de violence dont ont été victimes les femmes autochtones au Canada au cours des années 1970 et 1980, à savoir des disparitions forcées de nouveau-nés autochtones et des cas de stérilisation forcée ${ }^{33}$. Comme mentionné dans notre introduction, le rapport final de l'ENFFADA conclut pour la première fois à un génocide ${ }^{34}$, à la place du terme de " féminicide ", afin d'insister sur la dimension intersectionnelle de ces disparitions et assassinats : « Ce rapport porte sur un génocide planifié, fondé sur la race, l'identité et le genre $»^{35}$.

Cette violence est d'ailleurs retranscrite dans Deer Woman par les dessins de Jonathan R. Thunder, à travers la vivacité des traits hachurés, les scènes d'agressions sexuelles et de ripostes ainsi que le choix du noir et blanc, qui participe de la gravité de ce qui y est narré. Elle permet de signifier cette colère, voire cette rage, mais également cette tristesse, cette mélancolie et cette détermination à faire en sorte que justice soit faite et que le mal soit réparé. Tous ces sentiments ressortent aussi des témoignages des femmes autochtones victimes ou proches de victimes de violences sexuelles au Canada. L'œuvre de la réalisatrice abénakise Kim Obomsawin, Ce silence qui tue (2018), transmet 
l'expérience de ces femmes de façon magistrale. Face aux failles et aux échecs successifs du système de justice canadien, auquel elles se sont remises un temps, des formes de résistance et de résilience nouvelles se créent et se manifestent au sein des familles de victimes et de groupes de femmes en particulier, qui s'attèlent désormais elles-mêmes à faire ce qui n'a pas été fait ${ }^{36}$ : rechercher les corps de leurs proches portées disparues, diffuser le portrait de ces femmes, développer des initiatives d'entraide qui travaillent à la guérison ${ }^{37}$.

Dans l'œuvre d'Elizabeth LaPensée, le personnage de Deer Woman apparaît comme une figure d'appât redoutable: si les hommes la touchent ou s'approchent d'une femme en situation de vulnérabilité, ils en paieront les conséquences. À l'aune du contexte actuel particulier des féminicides de femmes autochtones, le récit de cette super-hérö̈ne sert à la fois de mise en garde destinée aux hommes et de mise en valeur de la femme autochtone, capable de se défendre par elle-même. C'est précisément ce que revendique l'association Armed Sisters, partenaire de la bande dessinée Deer Woman.

\section{Une œuvre-guide en faveur d'une auto-défense autochtone (éco)féministe}

21 L'histoire coloniale liant les femmes autochtones et le Canada nourrit le développement d'une rhétorique et de discours fondés sur une analogie : celle du corps de la femme (autochtone) comme terre colonisée. En d'autres termes, un grand nombre d'artistes femmes autochtones et/ou militantes fondent leur argumentaire sur un point historique et un autre d'ordre plus symbolique ${ }^{38}$. Les premiers arrivants européens ont colonisé les terres et à mesure de leur avancée sur le territoire, ils ont aussi souillé les corps des femmes autochtones, en les considérant comme des butins de guerre ou en se les appropriant à travers des représentations stéréotypées de la femme autochtone ${ }^{39}$ : la Squaw (femme autochtone de faible condition dont l'abus n'en constitue pas vraiment un) et la Princesse Indienne (femme autochtone respectée dans l'organisation sociétale). Pour déconstruire et résister à ces représentations issues de la logique patriarcale sous-jacente aux rapports de domination entre colonisateurs et colonisés ${ }^{40}$, plusieurs artistes autochtones développent l'idée selon laquelle le corps de la femme n'est pas une terra nullius, c'est-à-dire une "terre sans maître $»^{41}$, mais dispose bien d'un propriétaire : la femme qui l'habite ${ }^{42}$.

Cette analogie entre la colonisation de la terre et la colonisation du corps conduit à interroger non seulement la dimension féministe d'œuvres comme Deer Woman, mais plus spécifiquement leur dimension éco-féministe. En 1995, l'activiste autochtone anishinabe Winona LaDuke déclare ainsi : «La Terre est notre Mère. D'elle nous tirons notre vie, et notre vie, et notre capacité à vivre. C'est notre responsabilité de prendre soin de notre mère, et en prenant soin de notre Mère, nous prenons soin de nous. Les femmes, toutes les femmes, sont la manifestation de la Terre Mère sous la forme humaine (...) $»^{43}$. Si la notion même d'éco-féminisme a d'abord été forgée par Françoise d'Eaubonne (1974), d'autres chercheurs ont par la suite proposé de redéfinir ou de repenser cette expression, comme Karren J. Warren, qui met en évidence les intersections de la domination des femmes avec celle de la nature (1998). Comme le souligne Catherine Larrère (2014) :

De cette domination conjointe et croisée (la nature est vue comme une femme, les

femmes sont assimilées à la nature), Karen J. Warren analyse le «cadre 
conceptuel ». Dans la série des dualismes qui caractérisent la pensée moderne (nature / culture, nature / société, femme / homme, passif / actif, objet / sujet, émotionnel / rationnel, matière / esprit), elle voit les étapes d'une logique de la domination: la distinction (qui sépare en deux parties opposées ce qui dans d'autres contextes culturels peut apparaître en continuité), la hiérarchisation, ou valorisation (une partie est réputée inférieure à l'autre), la subordination enfin (la partie inférieure peut être légitimement subordonnée ou exploitée par la partie supérieure). Les hommes, sujets rationnels, actifs, sont ainsi en droit de faire des femmes et de la nature les objets passifs de leur domination. Karen J. Warren en appelle à leur libération conjointe, à la fin de tous les «ismes» oppresseurs : sexisme, racisme, naturisme, notamment. intéressant en ce qu'il lie la culture, le genre et la nature (Wilson, 2005). Ce personnage apparait ainsi non seulement comme une femme qui se défend, mais aussi comme la nature elle-même qui se défend ${ }^{44}$, dans la mesure où ses pouvoirs sont certes en elle mais activés, réveillés, par sa transformation en cerf au moment où elle en a besoin c'est-à-dire en une espèce animale ou en tant tout cas une espèce qui n'est pas humaine. D'autre part, la femme se transforme en cerf, c'est-à-dire en un être vivant dont le sexe biologique est masculin, faisant ainsi du personnage de Deer Woman une sorte de synthèse du vivant réconciliant les espèces, les sexes et les genres de telle sorte que finalement celui-ci apparaisse comme le degré 0 de la différenciation interespèce, intersexe, intergenre.

Ainsi, la bande dessinée Deer Woman ne représente pas uniquement la réactualisation d'un mythe ou la dénonciation des féminicides en cours. Symbole de la résilience des femmes autochtones qui se reconstruisent en réponse aux tentatives de génocides dont elles ont été victimes et aux attaques sexuelles auxquelles elles sont confrontées au quotidien ${ }^{45}$, cette œuvre se présente également comme un manuel de survie, un guide d'empowerment à la portée de toutes ${ }^{46}$. En témoignent des représentations dans la bande dessinée de techniques d'auto-défense de l'organisation Arming Sisters Reawakening Warriors (« Armer les sœurs, réveiller les guerrières » en français), une association qui promeut l'autonomisation et la défense comme outils de guérison et de résilience.

Créée en 2013 par Patty (Stein) Stonefish qui avait réalisé les bienfaits de l'auto-

défense des femmes dans sa propre vie, et voulait que les autres fassent

l'expérience de cette émancipation. En tant que survivante d'une agression sexuelle, Stonefish considère que sa guérison est en grande partie liée au parcours qu'elle a accompli grâce aux arts martiaux et à l'auto-défense féminine ${ }^{47}$.

L'une des planches de Deer Woman contient ainsi plusieurs dessins didactiques à destination des femmes désireuses d'apprendre des gestes d'auto-défense salvateurs. Des indications techniques précises y figurent, telles que :

Coup de poing : frappez le visage, la gorge, ou la partie inférieure des jambes avec votre pouce à l'extérieur de votre poing maintenu relâché. Frappe: frappez l'entrejambe en bougeant vers le haut avec la paume de votre main. Coup dans les yeux : utilisez votre doigt ou votre pouce pour attraper une partie d'un œil. Claque sur les oreilles : frappez une oreille avec une main relâchée et légèrement incurvée. Morsure : mordez une petite surface de peau sur les mains, le cou, ou les joues ${ }^{48}$.

Patty Stonefish (fondatrice de l'association Arming Sisters) précise néanmoins dans Deer Woman que l'auto-défense féminine ne concerne pas seulement le fait de savoir se battre physiquement : "L'auto-défense des femmes n'est pas qu'une question de coups dans les yeux, de coups de poing dans la gorge, et de frappes. C'est une question 
d'émancipation qui réveille, d'amour de soi, de maitrise de son corps... Ou du moins ça devrait l'être $»^{49}$.

La super-héroïne de Deer Woman n'est pas seulement mise en scène en tant que combattante. Elle apparaît également comme un personnage résilient, qui trouve en elle la force de se dépasser et de se reconstruire. Le but de cette bande dessinée est d'aider les femmes ayant subi des agressions sexuelles à sortir de la position de victime afin de réapprendre à devenir les sujets de leurs vies, comme en témoignent ces quelques phrases prononcées par Deer Woman dans les dernières pages: «Et à la fin, quoi que nous ayons connu, nous nous retrouvons toujours ${ }^{50}$.

\section{Conclusion}

Voir dans le personnage de Deer Woman une figure emblématique d'une forme de féminisme autochtone dénonçant et luttant uniquement contre les violences sexuelles dont les femmes autochtones sont les proies nous apparait restrictif.

En rompant aussi avec la catégorisation binaire « être humain vs. animal », Deer Woman réactualise un mythe traditionnel autochtone laissé aux mains de réalisateurs de cinéma blancs à des fins de pur divertissement ${ }^{51}$. À travers cette bande dessinée, nous pouvons également percevoir un combat livré contre la domination masculine, à laquelle sont confrontées non seulement les femmes (autochtones), mais plus largement la nature dont elles dépendent (comme nous). En somme, nous pouvons voir dans le personnage de la jeune femme violée se transformant en Deer Woman une représentation métaphorique de la Terre qui, à travers deux de ses espèces (le cerf et l'être humain, qui est aussi un animal) lutte contre les hommes qui tentent de la soumettre et de l'exploiter. Nous pouvons lire dans l'image du corps violé de la femme une allégorie de la terre violée par la colonisation qui a tenté, par tous les moyens militaires, juridiques, biologiques - de vider le continent nord-américain en annihilant ses premiers habitants, et $a$ fortiori les femmes.

Super-héroïne éco-féministe, Deer Woman incarne une révolte à l'égard de toute domination patriarcale, sur laquelle repose l'économie capitaliste occidentale qui tente de s'approprier le corps des femmes (autochtones) à l'instar des terres, dont les ressources naturelles font plus que jamais l'objet de projets extractivistes désastreux pour l'environnement et les populations locales. Les exemples ne manquent pas, et les femmes autochtones sont en première ligne dans ces luttes qui, sur le terrain, s'illustrent dans des mouvements de résistance de grande ampleur. Cela a notamment été le cas en 2016, lors de la mobilisation contre la construction de l'oléoduc Dakota Access Pipeline par la société Energy Transfer Partners dans la réserve Sioux de Standing Rock (Dakota du Nord, États-Unis) ${ }^{52}$, et plus récemment en territoire Secwepemc (Colombie-Britannique, Canada) où Kanahus Manuel et d'autres femmes autochtones protestent contre l'extension de l'oléoduc Trans Mountain par la société Kinder Morgan ${ }^{53}$.

31 Anonymisée ainsi sous les traits d'une jeune femme que nous pourrions toutes être, la super-héroïne de Deer Woman n'appelle pas seulement à une auto-défense et une résilience féminines autochtones, mais à la prise de conscience et à l'action de toutes les femmes, ainsi qu'à la responsabilisation des hommes. 


\section{BIBLIOGRAPHIE}

Réclamer notre pouvoir et notre place : le rapport final de l'Enquête nationale sur les femmes et les filles autochtones disparues et assassinées, 03 juin 2019. Version intégrale accessible aux adresses URL : https://www.mmiwg-ffada.ca/wp-content/uploads/2019/06/Rapport-final-volume-1a-1.pdf ; https://www.mmiwg-ffada.ca/wp-content/uploads/2019/06/Rapport-final-volume-1b.pdf et https://www.mmiwg-ffada.ca/wp-content/uploads/2019/06/Rapport-

compl\%C3\%A9mentaire_Qu\%C3\%A9bec.pdf. Pages consultées le 06.06.2019.

ALLEN Paula Gunn (1991), Grandmothers of the Light: A Medicine Woman's handbook, Boston, Beacon Press.

ALLEN Paula Gunn (1992), The Sacred Hoop. Recovering the Feminine in American Indian Traditions, Boston, Beacon Press.

APPADURAI Arjun (2001), Après le colonialisme. Les conséquences culturelles de la globalisation, Paris, Petite Bibliothèque Payot.

BERLO Janet Catherine (1993), « Dreaming of Double Woman: The Ambivalent Role of the Female Artist in North American Indian Myth », American Indian Quaterly, vol. 17, n 1.

CHAVARRIA Tony (2009), «Indigenous Comics in the United States », World Literature Today, vol. 83, $\mathrm{n}^{\circ} 3$, p. 47-49.

CHONG Bras Andrea (2017), « Deer Woman: An Anthology' Sheds Light on Violence Against Native Women in North America».

Source URL : https://rising.globalvoices.org/blog/2017/12/15/deer-woman-an-anthology-shedslight-on-violence-against-native-women-in-north-america/. [Page consultée le 05.12.2018].

FITZGERALD Tanya (2006), « Walking between Two Worlds: Indigenous Women and Educational Leadership ", Educational Management Administration \& Leadership, vol. 34, n² 2, p. 201-213.

FRAISSE Geneviève (2012), La Fabrique du féminisme. Textes et entretiens, Le Passager Clandestin, coll. « Essais».

GILBERT Samuel (2016), « Native Superheroes Battle Old Stereotypes at the First Ever Indigenous Comic Con », Vice (en ligne), publié le 26.11.2016. Source URL : https://www.vice.com/en_us/ article/jmkv88/native-superheros-battle-old-stereotypes-at-the-first-ever-indigenous-comiccon. [Page consultée le 03.12.2018].

HACHE Emilie (textes choisis et présentés par) (2016), RECLAIM. Anthologie de textes écoféministes, traduit de l'anglais par Emilie Noteris, Paris, Editions Cambourakis.

KUOKKANEN Rauna (2007), « Myths and Realities of Sami Women. A Post-Colonial Feminist Analysis for the Decolonization and Transformation of Sami Society », in Joyce Green (dir.), Making Space for Indigenous Feminism. Black Point/Winnipeg, Fernwood Pub, p. 72-92.

LAINÉ Jean-Marc (2011), Super-héros ! La puissance des masques, Lyon, Moutons électriques.

LAPENSÉE Elizabeth (et al.) (2015), Deer Woman: A Vignette, Albuquerque, Native Realities Press.

LAPENSÉE Elizabeth (et al.) (2017), Deer Woman: An Anthology, Albuquerque, Native Realities Press. 
LARRÈRE Catherine (2014), «L'écoféminisme : féminisme écologique ou écologie féministe », Tracés. Revue de Sciences humaines [En ligne], 22 | 2012. [Page consultée le 08.04.2019]. Source URL : http:// journals.openedition.org/traces/5454.

MAUZÉ Marie (2018), « Un mangaka en pays haïda », blog de la revue Carnets de Terrain. Source URL : https://blogterrain.hypotheses.org/10265. [Page consultée le 05.12.2018].

OLIVER Kelly (2016), Hunting Girls. Sexual Violence from The Hunger Games to Campus Rape, New York, Columbia University Press.

PERREAULT Julie (2015), «La violence intersectionnelle dans la pensée féministe autochtone », Recherches féministes, vol. 28, no 2, p. 33-52.

POWERS Marla N. (1980), « Menstruation and Reproduction: An Oglala Case », Signs, vol. 6, n 1, Women: Sex and Sexuality, p. 54-65.

POWERS William K. (1977), Oglala Religion, Lincoln, University of Nebraska Press.

WALTER Emmanuelle (2014), préface de Widia Larivière, Soeurs Volées. Enquête sur un féminicide au Canada, Montréal, Lux Editeur.

WARREN Karen J. (1998) [1990], « The power and the promise of ecological feminism », Environmental Philosophy. From Animal Rights to Radical Ecology, M. Zimmerman éd., New Jersey, Prentice Hall, p. 325-345.

ZECHOWSKI Sharon et Neumann Caryn E. (2014), « The Mother of All Superheroes: Idealizations of Femininity in Wonder Woman » dans Heroines of comic books and literature: portrayals in popular culture, Lanham etc., Etats-Unis d'Amérique, Rowman \& Littlefield, p. 133-144.

zoLBRoD Paul G. (1984), Dine Bahane': The Navajo Creation Story, Albuquerque, University of New Mexico Press.

\section{NOTES}

1. Paula Gunn Allen, The Sacred Hoop. Recovering the Feminine in American Indian Traditions, Beacon Press, Boston, 1992, p. 189.

2. Marvel s'illustre notamment en 2015 en consacrant un livre entier à son personnage Red Wolf, représenté à de nombreuses reprises depuis 1976, faisant de lui le premier super-héros autochtone dans le monde de la bande dessinée. En 2018, la même maison d'édition décide de mettre en scène dans le numéro 19 de sa série Champion une héroïne autochtone, Amka Aliyak, alias Snowguard, jeune lycéenne Inuit capable de se transformer en animal.

3. À ce sujet, voir notamment: Tony Chavarria, «Indigenous Comics in the United States ", in World Literature Today, vol. 83, nº 3 (May - Jun., 2009), p. 47-49.

4. À ce sujet, voir notamment le très bon article du média alternatif Vice: Samuel Gilbert, « Native Superheroes Battle Old Stereotypes at the First Ever Indigenous Comic Con », in Vice (en ligne), publié le 26.11.2016. Source URL: https://www.vice.com/en_us/article/jmkv88/nativesuperheros-battle-old-stereotypes-at-the-first-ever-indigenous-comic-con. Page consultée le 03.12.2018.

5. Elizabeth LaPensée est Anishinaabe et Métis d'origine irlandaise; au Canada, les peuples autochtones reconnus sont les Premières Nations, les Métis, et les Inuits.

6. Jonathan R. Thunder est Anishinaabe.

7. Weshoyot Altrive est une artiste Tongva et gaélique écossaise. 
8. Qui reprend intégralement Deer Woman: A Vignette, d'Elizabeth LaPensée et Jonathan R. Thunder.

9. Si notre étude porte davantage sur les féminicides auxquelles sont confrontées les femmes autochtones au Canada, nous avons mentionné également les États-Unis dans notre titre dans la mesure où ce fléau concerne aussi les femmes autochtones des États-Unis. Les données statistiques sont néanmoins beaucoup moins précises et accessibles, ces situations ne faisant pas l'objet de véritables enquêtes de la part des instances judiciaires états-uniennes.

10. Cette expression nous est inspirée par l'article de Fitzgerald, T. (2006). «Walking between Two Worlds: Indigenous Women and Educational Leadership ", in Educational Management Administration \& Leadership, vol. 34, $\mathrm{n}^{\circ}$ 2, p. 201-213.

11. Les féminicides ont des conséquences sociales sur les rapports qu'entretiennent les femmes autochtones à leurs identités et à leurs familles; ils sont aussi un problème sociétal dans la mesure où la société canadienne a éludé ce phénomène jusqu'à tout récemment et n'a commencé à l'évoquer qu'après la Commission Vérité et Réconciliation de 2014.

12. Terminologie utilisée afin de désigner les assassinats de femmes commis parce qu'elles sont des femmes. Aux États-Unis et au Canada, les premières victimes des féminicides sont les femmes autochtones, qui combinent deux à trois facteurs de vulnérabilité: le fait d'être femme, autochtone, et de classe sociale pauvre.

13. Intitulé « Réclamer notre pouvoir et notre place : le rapport final de l'Enquête nationale sur les femmes et les filles autochtones disparues et assassinées ", sa version intégrale est accessible aux adresses URL: https://www.mmiwg-ffada.ca/wp-content/uploads/2019/06/Rapport-finalvolume-1a-1.pdf ; https://www.mmiwg-ffada.ca/wp-content/uploads/2019/06/Rapport-finalvolume-1b.pdf et https://www.mmiwg-ffada.ca/wp-content/uploads/2019/06/Rapportcompl\%C3\%A9mentaire_Qu\%C3\%A9bec.pdf. Pages consultées le 06.06.2019.

14. D'autres auteures font la distinction entre "patriarcat » et "fratriarcat », qui serait plus en adéquation avec les réalités actuelles. C'est notamment le cas de l'universitaire Geneviève Fraisse, qui déclare : "Dans nos sociétés occidentales, nous ne sommes plus dans le patriarcat puisque nous avons effectivement travaillé pendant ces deux derniers siècles à produire une multiplication de possibilités égalitaires dans l'espace public comme dans l'espace privé. Le patriarcat peut rester dans nos têtes, mais il n'y a plus de chef de famille. Il n'y en a plus au niveau des lois, même si dans l'administration il y a encore des comportements qui relèvent du patriarcat; mais ce n'est pas légal. Le patriarcat est un mot pour dire la domination masculine, mais le père chef de famille n'existe plus ». Cf. La Fabrique du féminisme. Textes et entretiens, Le Passager Clandestin, coll. "Essais », 2012, p.390. Par ailleurs, les théories écoféministes ont été développées par d'autres auteures et nous renvoyons en ce sens à l'anthologie de textes féministes RECLAIM. Anthologie de textes écoféministes (choisis et présentés par Emile Hache), traduit de l'anglais par Emilie Noteris, Paris, Editions Cambourakis, 2016.

15. Thèse en préparation à l'École des Hautes Études en Sciences Sociales depuis 2015, sous la direction de Marie Mauzé (CNRS) et la codirection de Michel Poivert (Université Paris 1 Panthéon-Sorbonne) intitulée « Artistes femmes et queer autochtones en Amérique du Nord face à leur(s) image(s), des années 1970 à nos jours. Une histoire intersectionnelle et décoloniale des arts contemporains autochtones aux États-Unis et au Canada ».

16. Nous utilisons le terme "écoféministe" afin de qualifier un personnage ou une personne dont les représentations et les actes traduisent des revendications qui visent à soustraire du patriarcat et de la domination masculine tant les femmes que la nature.

17. Cet anglicisme trouve son origine dans le mot «Indigenization » dont a notamment fait usage Arjun Appadurai dans son ouvrage paru pour la première fois en 1996 sous le titre Modernity at Large. Cultural Dimensions of Globalization. Pour une traduction française, voir: Arjun Appadurai, Après le colonialisme. Les conséquences culturelles de la globalisation, Petite Bibliothèque Payot, Paris, 2001. 
18. "My paintings explore personal themes of identity and internal dialogue through story and characters. I depict expressive characters whose emotions and thoughts manifest viscerally in their physical form. The bodies of my subjects often appear fragmented, animalistic, or partially obscured. My art acts as the scrapbook recording an evolving identity. Through my subjects, I can exaggerate the villains and heroes that make up my self-image or the worldview as seen through my lens. My cultural identity infuses with my identity as an urban dweller. These paintings are vignettes by nature, a glimpse at a moment in a story that neither begins nor ends on the canvas". Traduit de l'anglais au français par l'auteure. Source URL: http:// www.mnartists.org/jonathanrthunder. Page consultée le 24.04.2019.

19. Voir par exemple : Jonathan R. Thunder, Deer Woman Gets A Manicure, peinture acrylique sur toile, 54"x48", 2016.

20. « The Deer Woman story has been told in my family as a frightening tale. Here I bring beauty and femininity to the classic character ». Traduit de l'anglais au français par l'auteure. Source URL : http://www.mnartists.org/artwork/deer-woman-gets-manicure. Page consultée le 24.04.2019.

21. William K. Powers, Oglala Religion, Lincoln : University of Nebraska Press, 1977, p. 197. Cité par Maria N. Powers, «Menstruation and Reproduction: An Oglala Case », in Signs, Vol. 6, No. 1, Women: Sex and Sexuality, Part 2 (Autumn, 1980), p. 62.

22. Marla N. Powers, op. cit., p. 61-62 : «Another example of female symbolism is found in a mythological character called Anukite, who plays an important role in the supernatural affairs of the Oglala. In the cosmology Ite (Face) is very beautiful and is married to Tate (the Wind). Ite has an affair with the Sun, who at the time is married to the Moon. Because of her infidelity Ite is punished and given an ugly face. On one side she is still beautiful, on the other ugly. She thus becomes Anukite (Double Face), who, according to Powers, "appears to men in visions and in the real world in the form of a deer or two deer women, one white and the other black. The two faces of Double Face and the two Deer Women represent proper and improper sexual conduct.... Men become disorderly or crazy when they have [improper] sexual relations with deer women." Women who dream of Anukite have unusual powers to seduce men. These women were considered wakan (sacred). The Oglala say that if a man meets a lone woman in the woods or on the prairie he must avoid her, for she may be a deer woman. The myth attributes to the deer a peculiar scent in the hoof that becomes a fine perfume when the deer becomes a woman. The perfume then acts as a medicine and works an evil spell on men. Sometimes even wishing to make love to a deer woman can be fatal. Deer women often appear as beautiful creatures who, after enticing men to have improper sexual relations with them, turn into deer and run away. After this the man goes insane or dies. » Traduit de l'anglais au français par l'auteure, comme les citations suivantes.

23. À ce sujet, voir notamment l'ouvrage de Paula Gunn Allen, op. cit.

24. Zolbrod Paul G., Dine Bahane': The Navajo Creation Story, University of New Mexico Press, 1984.

25. Weshoyot Altrive in Chong Bras Andrea, 'Deer Woman: An Anthology' Sheds Light on Violence Against Native Women in North America. Mis en ligne le 16 décembre 2017. Source URL : https://rising.globalvoices.org/blog/2017/12/15/deer-woman-an-anthology-sheds-light-onviolence-against-native-women-in-north-america/. Page consultée le 05.12.2018. « There is a lot of hurt and a lot of broken feminine ideals in Native culture, especially currently. And I am hoping with what I've done with the book [...], is to take that broken lost figure, who is almost mythological, and have her deal with modern issues. So it touches real issues and touches real Native women ".

26. À ce sujet, voir notamment: Allen Paula Gunn, Grandmothers of the Light: A Medicine Woman's handbook, Boston, Beacon Press, 1991. 
27. Au Canada, cette véritable pandémie de disparitions et d'homicides dont sont victimes les femmes autochtones fait d'ailleurs l'objet d'une "Commission d'enquête sur les femmes disparues et assassinées", dont les résultats sont attendus pour le mois d'avril 2019. À ce jour, Fanny Wilde (première avocate algonquine du Québec) révèle que la commission a réuni plus de 2000 témoignages. Propos recueillis à l'occasion de la conférence internationale donnée à l'INALCO (Paris), le 10 décembre 2018, «La Voix des Femmes Autochtones », organisée par l'association En Terre Indigène. Captation vidéo de l'événement accessible à l'adresse suivante : https://www.facebook.com/FemmesAutochtones/videos/1023270194531871/?hc_location=ufi.

28. C'est ce que souligne notamment Andrea Chong Bras, op. cit.

29. Elizabeth LaPensée, Jonathan R. Thunder, op. cit., p. 1: «This is a call to awareness to recognize that there are over 1,000 missing and murdered Indigenous women of all ages. It is situated along the Great Lakes where young women and many others are involved in sex trafficking via boats. It calls us to take a stance to change the fact that Indigenous women are the most likely to experience sexual assault on Turtle Island and to recognize the lack of rights (...) I hope for more of deer woman's stories to be told, for her to be heard, and most of all, for Indigenous Women and our families and communities to heal ».

30. «In the United States, violence against indigenous women has reached unprecedented levels on tribal lands and in Alaska Native villages. More than 4 in 5 American Indian and Alaska Native women have experienced violence, and more than 1 in 2 have experienced sexual violence. Alaska Native women continue to suffer the highest rate of forcible sexual assault and have reported rates of domestic violence up to 10 times higher than in the rest of the United States. Though available data is limited, the number of missing and murdered American Indian and Alaska Native women and the lack of a diligent and adequate federal response is extremely alarming to indigenous women, tribal governments, and communities. On some reservations, indigenous women are murdered at more than ten times the national average ". Traduit de l'anglais au français par l'auteure. Source URL : https://indianlaw.org/issue/ending-violenceagainst-native-women. Page consultée le 17.10.2018.

31. Emmanuelle Walter, préface de Widia Larivière, Soeurs Volées. Enquête sur un féminicide au Canada, Lux Editeur, 2014, p. 11 et 14.

32. Créée à la suite de la Commission de Vérité et Réconciliation canadienne, dont le rapport final a été rendu en 2015, et qui avait pour principal objet de recueillir les témoignages des victimes abusées physiquement et sexuellement dans les pensionnats indiens dans lesquels les enfants autochtones étaient envoyés jusqu'à la fin des années 1980 .

33. Propos recueillis lors de la conférence internationale organisée à l'INALCO (Paris), le 10.12.2018, « La Voix des Femmes Autochtones », op. cit.

34. Terme utilisé près de 170 fois au fil des 1000 pages du rapport.

35. Op. cit., 2019, p. 7.

36. C'est notamment ce que fait l'association Native Women's Association of Canada et son programme « Sisters in Spirit Initiative » lancé en 2005.

37. La dénonciation des féminicides de femmes autochtones aux États-Unis et au Canada passe également par d'autres formes artistiques, telles que des performances et des installations, qui font appel à un motif récurrent: la robe rouge. Ainsi, un grand nombre d'œuvres présentent cette iconographie, en optant pour une représentation dans laquelle le corps est au centre et incarne de manière personnelle la disparition de ces femmes sous la forme d'une célébration, d'un hommage, ou au contraire en choisissant de mettre l'accent sur l'esprit par l'absence du corps, caractéristique de la disparition. Voir notamment la performance de Rebecca Belmore (Anishinaabe), Vigil, 2002 et l'installation de Jaime Black (Métis), The RedDress Project, 2014.

38. À ce sujet, voir par exemple l'oeuvre d'Amanda Lickers (Turtle Clan, Seneca) et Lindsay Nixon (Nehiyaw / Anishinaabe), « Terra Nullius Is Rape Culture », dessin noir et blanc et broderie au fil rouge ; ou encore Erin Marie Konsmo (Métis / Cree), « Geographic Racism », 2013, dessin couleur. 
39. Nous pensons notamment aux œuvres des artistes Wendy Red Star (Crow), Erica Lord (Inupiaq / Athabascan), et Lori Blondeau (Anishinaabe).

40. À ce sujet, nous renvoyons aux travaux de Julie Perreault (Université d'Ottawa), et plus particulièrement à son article «La violence intersectionnelle dans la pensée féministe autochtone ", Recherches féministes, vol. 28, no 2, 2015, p. 33-52, dans lequel elle cite Rauna Kuokkanen (Sami) : «Je crois fermement que notre survie en tant que peuple dépend de la manière dont nous transformerons et décoloniserons le discours colonial et patriarcal qui se réverbère dans tous les aspects de nos sociétés. Celui-ci nous distrait et nous empêche de restaurer et d'imaginer à nouveau nos communautés et l'avenir de nos peuples. Le processus de décolonisation doit contester la fondation de l'ordre social et culturel prescrit par les systèmes coloniaux et patriarcaux, c'est-à-dire s'attaquer à l'intersection des oppressions et des mécanismes de pouvoir à des niveaux institutionnels et structurels» (Kuokkanen, 2007 : 85).

41. La notion de «terra nullius » ou du «territoire sans maître », remonte à la bulle papale Terra Nullius du pape Urbain II (1095). À ce sujet, voir notamment : Pramod K. Nayar, The Postcolonial Studies Dictionary, p. 153. Il explique notamment: «Dérivé du latin, cela signifie littéralement «terre qui n'appartient à personne ». Le terme provient de la bulle papale Terra Nullius formulée par le Pape Urbain II en 1095, au début des Croisades. La Bulle autorisait les princes et les rois européens à « découvrir » ou revendiquer n'importe quelle terre occupée par des personnes nonchrétiennes dans n'importe quelle partie du monde alors connu ou du monde appelé à être connu. En 1452, cette règle a été étendue par le Pape Nicolas V. Le terme signifie alors possession et propriété du territoire en faisant la distinction entre les chrétiens qui sont en droit de posséder la terre et les barbares/non-chrétiens qui ne le sont pas. Cette Bulle a conduit à ce qui est désormais connue comme la Doctrine de la Découverte, et constitue l'une des idées (et idéologies) qui ont inspiré l'expédition de Colomb en 1492. Cela peut aussi être vu comme le motif directeur derrière la "thèse de la frontière » et de l'expansion vers l'Ouest du $19^{\text {ème }}$ siècle en Amérique du Nord : puisque les autochtones vivaient à " l'état de nature " ils ne pouvaient pas prétendre à posséder la terre - c'est ce qu'a mis en pratique l'attitude de Thomas Jefferson à l'égard des autochtones ». Traduit de l'anglais au français par l'auteure. Citation originale: «Derived from Latin, it literally means 'land that belongs to no one'. The term comes from the Papal Bull Terra Nullius issued by Pope Urban II in 1095, at the beginning of the Crusades. The Bull allowed Europeans princes and kings to 'discover' or claim any land occupied by nonChristian peoples in any part of the then known and to-be-known world. In 1452 this policy was extended by Pope Nicholas V. The term signifies possession and ownership of territory by distinguishing between Christians who are entitled to own land and barbarians/non-Christians who are not. This Bull led to what came to be known as the Doctrine of Discovery, and was one of the inspirational ideas (and ideologies) behind Columbus' expedition in 1492. It could also be treated as the driving motif behind the 'frontier thesis' and the Westward expansion of the $19^{\text {th }}$ century in North America : since the Native Americans were living in a 'state of nature' they could not be deemed to own the land - an attitude exemplified in Thomas Jefferson's attitude toward the natives ».

42. À ce sujet, voir par exemple PJ Lilley et Erin Marie Konsmo, «'Art Through a Birch Bark Heart': An Illustrated Interview », in Radical Criminology. An Insurgent Journal, 2, 2013 ; et plus spécifiquement l'œuvre graphique d'Erin Marie Konsmo, Our Bodies Are Not Terra Nullius.

43. «The Earth is our Mother. From her we get our life, and our life, and our ability to live. It is our responsibility to care for our mother, and in caring for our Mother, we care for ourselves. Women, all females, are the manifestation of Mother Earth in human form. We are her daughters and in my cultural instructions: Minobimaatisiiwin. We are to care for her. I am taught to live in respect for Mother Earth. In Indigenous societies, we are told that Natural Law is the highest law, higher than the law made by nations, states, municipalities and the World Bank. That one would do well to live in accordance with Natural Law. With those of our Mother. And in respect for our 
Mother Earth of our relations -- indinawaymuguni took». Traduit de l'anglais au français par l'auteure. Source URL : https://ratical.org/co-globalize/WinonaLaDuke/Beijing95.html. Page consultée le 28 août 2018.

44. «Nous ne défendons pas la nature, nous sommes la nature qui se défend » est un slogan qui a notamment été utilisé par les opposant.e.s au projet d'aéroport à Notre-Dame-des-Landes.

45. Voir Paula Gunn Allen, op. cit., « Angry Women Are Building: Issues and Struggles Facing American Indian Today », p. 189.

46. Notons que cette bande dessinée peut être acquise sur le site de la maison d'édition Native Realities Press gratuitement. Elle est téléchargeable à l'adresse suivante: téléchargeable à l'adresse suivante : https://www.nativerealities.com/products/deer-woman-a-vignette.

47. « Founded 2013 by Patty (Stein) Stonefish upon realizing the healing benefits of women's selfdefense in her own life \& wanted others to experience the same sense of self-empowerment. As a survivor of sexual assault, Stonefish credits a large part of her healing to her journey with martial arts \& women's self-defense ». Traduit de l'anglais au français par l'auteure. Source URL : https://armingsisters.org/about. Page consultée le 06.12.2018.

48. Elizabeth LaPensée, Jonathan R. Thunder, op. cit., p. 20 : «Punch: Punch the face, throat, or lower legs with your thumb outside your fist which is held loose. Going Strike: Hit the groin moving upwards with the heel of your hand. Eye gouging: Use your finger or thumb to scrape part of an eye. Ear slap: Strike an ear with a loose and slightly cupped hand. Bite: Bite a moderate amount of skin at the hands, neck, or cheeks. »

49. Patty Stonefish, in Elizabeth LaPensée, op. cit., p. 21 : «Women's self defense isn't all about eye gouging, throat punches, and striking. It's about reawakening empowerment, self-love, and ownership of body... Or at least it should be ».

50. Elizabeth LaPensée, Jonathan R. Thunder, op. cit., p. 19 : « And in the end, whatever we have experienced, we always returned to ourselves ".

51. Voir notamment Masters of Horror: Deer Woman (John Landis, 2005).

52. Ce mouvement historique a réuni plus de 200 tribus autochtones différentes ainsi que de nombreux soutiens internationaux.

53. Kanahus Manuel a initié le mouvement des «Tiny Warrior Houses ", qui consiste à ériger des petites maisons mobiles et écologiques le long du tracé de l'oléoduc afin d'empêcher son extension effective.

\section{RÉSUMÉS}

À travers l'analyse de la bande dessinée Deer Woman créée en 2015 par Elizabeth LaPensée (Anishinaabe, Canada), notre article a pour objectif principal d'interroger la notion de "superhéroïne " à l'aune de représentations autochtones engagées. Il s'agit de voir en quoi cette revitalisation du mythe traditionnel de la Femme Cerf (Deer Woman) interroge l'identité en termes de sexe et de genre, la culture et la place des femmes autochtones, en prenant une position radicale face au problème sociétal des féminicides en Amérique du Nord. Nous postulons que la figure de Deer Woman peut aussi être vue aujourd'hui comme une allégorie mettant en garde contre la domination masculine sur les femmes et sur toutes autres formes de vie. Tout d'abord, nous étudierons les caractéristiques formelles de Deer Woman et les mythes fondateurs autochtones qui en sont à l'origine. Nous montrerons ensuite en quoi cette super-héroïne dont les pouvoirs apparaissent après qu'elle a été sexuellement agressée, tend à dénoncer et lutter 
contre les féminicides touchant les femmes autochtones. Enfin, nous nous demanderons comment cette bande dessinée pourrait laisser entrevoir l'émergence d'une super-héroïne autochtone éco-féministe.

Through the analysis of the comic book Deer Woman created in 2015 by Elizabeth LaPensée (Anishinaabe, Canada), our paper aims to question the notion of « super-woman » on the basis of involved Native American depictions. Our goal is to see how this revitalization of the traditional story of Deer Woman deals with Indigenous women's identity, cultural, and role issues, by taking a stand against the societal problem of feminicides in North America. We argue that Deer Woman is a character that can be seen today as an allegory that warns against men domination over women and any other kind of life. First of all, we will study the formal characteristics of Deer Woman and the Native American mythological basis upon which this comic is builded. Then, we will show how this super-woman, who gets her powers after having been sexually assaulted, tries to denounce and fight against feminicides against Indigenous women. Finally, we will ask how this comic book could let us think that we assist to the birth of an eco-feminist Indigenous superwoman.

\section{INDEX}

Mots-clés : super-hérö̈ne, bande dessinée, autochtone, féminicides, Amérique du Nord Keywords : superwoman, comic books, indigenous, feminicides, north america

\section{AUTEUR}

\section{AURÉLIE JOURNÉE-DUEZ}

Aurélie Journée-Duez, diplômée de l'Université Paris 1 Panthéon-Sorbonne et de l'Ecole du Louvre, est doctorante en anthropologie sociale et ethnologie à l'EHESS (LAS) sous la direction de Marie Mauzé (CNRS), et en histoire de l'art sous la codirection de Michel Poivert (Université Paris 1). Elle prépare une thèse intitulée « Artistes femmes, queer et autochtones face à leur(s) image(s). Pour une histoire intersectionnelle et décoloniale des arts contemporains autochtones aux États-Unis et au Canada, des années 1970 à nos jours ». Elle a récemment contribué à la version anglaise de l'ouvrage collectif Sexe, Race \& Colonies à paraître début 2020. Son dernier article se consacre à la réoccupation autochtone de l'espace urbain par l'art dans le cadre de mobilisations collectives contre l'extractivisme. Elle est par ailleurs présidente du Comité de Solidarité avec les Indiens des Amériques (CSIA-Nitassinan), association fondée en 1978 qui œuvre à diffuser les revendications des peuples autochtones et à promouvoir leurs arts et leurs cultures en France. 